\title{
Autoeficacia académica y las barreras para la búsqueda de ayuda para problemas de salud mental en Estudiantes Universitarios de Lima
}

\author{
Academic self-efficacy and its relation to the barriers to seeking help for mental health problems in \\ University Students of Lima
}

Alberto Salvador Palacios Jimenez ${ }^{1}$

\section{RESUMEN}

Objetivos: Determinar la relación entre la autoeficacia académica y las barreras para la búsqueda de ayuda para problemas de salud mental en estudiantes de una universidad pública de Lima. Materiales y métodos: El presente, es un estudio cuantitativo, no experimental, de tipo transversal. La muestra estuvo constituida por 131 estudiantes de una universidad pública de Lima. Los instrumentos utilizados fueron la Escala de autoeficacia percibida en situaciones académicas y el Cuestionario de barreras para la búsqueda de ayuda para problemas de salud mental. Resultados: En cuanto a la Autoeficacia académica, 40 estudiantes $(30,53 \%)$ presentaron un nivel alto de autoeficacia, $67(51,15 \%)$ obtuvieron un nivel regular y $24(18,32 \%)$ obtuvieron un bajo nivel. Con respecto a las barreras para la búsqueda de ayuda para problemas de salud mental, 67 estudiantes $(51,15 \%)$ obtuvieron una alta presencia de barreras, $31(23,66 \%)$ obtuvieron una presencia regular de barreras y $33(25,19 \%)$ obtuvieron una presencia baja de barreras. Conclusiones: Existe una relación significativa inversamente proporcional moderada entre la autoeficacia académica y las barreras para la búsqueda de ayuda para problemas de salud mental en un grupo de estudiantes de una universidad pública de Lima.

Palabras clave: autoeficacia académica, salud mental, estudiantes universitarios.

\begin{abstract}
Objectives: To determine the relationship between academic self-efficacy and the barriers to seeking help for mental health problems in students of a public university in Lima. Materials and methods: The present is a quantitative, not experimental, cross-sectional study. The sample consisted of 131 students from a public university of Lima. The instruments used were the Scale of perceived self-efficacy in academic situations and the Barriers Questionnaire for seeking help for mental health problems. Results: Regarding Academic Self-efficacy, 40 students $(30.53 \%)$ presented a high level of self-efficacy, $67(51.15 \%)$ obtained a regular level and $24(18.32 \%)$ obtained a low level. With respect to barriers to seeking help for mental health problems, 67 students $(51.15 \%)$ had a high presence of barriers, $31(23.66 \%)$ had a regular presence of barriers and 33 (25.19). \%) obtained a low presence of barriers. Conclusions: There is a significant, moderate, inversely proportional relationship between academic self-efficacy and barriers to seeking help for mental health problems in a group of students at a public university in Lima.
\end{abstract}

Keywords: academic self-efficacy, mental health, university students.

${ }^{1}$ EP de Educación. Universidad Nacional Mayor de San Marcos. Lima - Perú

\section{INTRODUCCIÓN}

Algunos de los factores más importantes para el desarrollo de las potencialidades del individuo durante su proceso formativo son los intrínsecos, aquellos que tienen que ver con la emocionalidad de la persona y sus juicios de valor sobre su entorno y, de manera especial, sobre sí mismo.
La autoeficacia académica es el conjunto de juicios de un individuo para manejar y afrontar acciones en el ámbito académico (1). Bandura, quien planteara por primera vez este constructo, definía la autoeficacia académica como la confianza en las propias capacidades intelectuales, las mismas que tienen como fin desarrollar habilidades cognitivas (2), en otras 
palabras, la autoeficacia académica es un conjunto de ideas sobre habilidades en el desarrollo académico personal, sin embargo, estas habilidades no están necesariamente reflejadas en la realidad (3).

Está demostrado que la autoeficacia académica, sumado al impulso de algunas conductas específicas, se desempeña como un factor protector ante el estrés académico (4), se asocia con la motivación y la creación de incentivos para alcanzar metas y promueve la aparición de actitudes entre las que están: el fortalecimiento de algunas ideas gracias a las experiencias de dominio (derivadas de las experiencias de éxito/fracaso), los aprendizajes obtenidos a partir de las experiencias de otros individuos, la persuasión verbal a otros individuos $\mathrm{y}$ algunos estados fisiológicos que tienen que ver con el afrontamiento (5).

En el Perú, el acceso a información sobre el estado de la salud mental de la población es escasa, sin embargo, la búsqueda de ayuda oportuna para problemas de salud mental es un determinante importante para procurar el bienestar propio. Para el presente estudio, las "barreras" son aquellas situaciones que inhiben el servicio de consejería o atención por parte de profesionales de la salud mental (6).

A su vez, evitar buscar ayuda de un profesional ante problemas de salud mental tiene que ver con factores como los relacionados específicamente con el trastorno, la preocupación por el estigma, la incomodidad de revelarlo, el miedo a que los síntomas empeores, la falta de confianza en los profesionales de la salud mental o en los tratamientos, el inmediatismo, las opiniones de terceros, factores relacionados a la condición económica o, en algunos casos, la determinación de manejar por cuenta propia el problema (7).

De la misma forma, es importante recalcar que este rechazo a la ayuda en problemas de salud mental repercute en un agravante a la condición de la persona, posiblemente a una larga lista de problemas de salud mental como consecuencia de no tratar el original o, se corre mayor riesgo de sufrir una recaída una vez empezado el tratamiento (6).

Viendo que algunas actitudes personales guardaran relación con las barreras para la búsqueda de ayuda para problemas de salud mental, el presente estudio busca determinar la relación que pudiera existir entre la autoeficacia académica y las barreras para la búsqueda de ayuda para problemas de salud mental en un grupo de estudiantes de una universidad pública de Lima. Así mismo, se busca determinar la relación entre los componentes de la variable autoeficacia académica y las barreras para la búsqueda de ayuda para problemas de salud mental.

\section{MATERIALES Y MÉTODOS}

El presente estudio es de enfoque cuantitativo, de diseño no experimental, de tipo descriptivo y corte transversal. La muestra de estudio es de naturaleza no probabilística, seleccionada por acceso. Corresponde a 131 estudiantes universitarios de la Escuela de Estudios Generales de una universidad pública de Lima (Perú). En la muestra prevalece el sexo femenino $(57,3 \%)$ sobre el masculino (42,7\%). Las edades están entre los 16 y 28 años, con una edad media de 19,37 y una desviación típica de 1,947 .

Para la recolección de los datos se utilizaron dos instrumentos: El primer instrumento es la Escala de autoeficacia percibida en situaciones académicas (EACA) compuesta por 13 ítems de respuesta cerrada en escala de Likert ( $1=$ Nunca; $5=$ Siempre) que valoran las dimensiones Comunicación (4 ítems), Atención (5 ítems) y Excelencia (4 ítems) (7). Un puntaje más indica un mayor grado de autoeficacia académica. El segundo instrumento es una adaptación del Cuestionario de barreras para la búsqueda de ayuda para problemas de salud mental, versión breve $(B A S H-B) \quad(6,8)$. El cuestionario es unifactorial y se compone de 10 ítems de respuesta cerrada en escala de Likert $(1=$ altamente en desacuerdo; 6 = altamente de acuerdo) que evalúan las barreras personales para la búsqueda de ayuda profesional. Un mayor puntaje indica la percepción de más barreras para la búsqueda de ayuda para problemas de salud mental.

Para el procesamiento de los datos se utilizó el software estadístico Statistical Package for the Social Sciences (IBM-SPSS, versión 23.0). Los procedimientos utilizados fueron: el análisis de validez factorial de los instrumentos, el análisis de la consistencia interna para determinar la confiabilidad, el análisis de las medidas de tendencia central, la estimación de la normalidad de la distribución de los resultados y el cálculo de los coeficientes de correlación entre las variables y sus componentes.

\section{RESULTADOS Y DISCUSIÓN}

Las propiedades psicométricas de los instrumentos se determinaron en función a las pruebas Medida de adecuación muestral KaiserMeyer-Olkin (KMO) y Medida de esfericidad de 
Bartlett para la validez y el Alfa de Cronbach para la confiabilidad.

El análisis factorial de la Escala de autoeficacia percibida en situaciones académicas (EACA) obtuvo un índice de adecuación muestral de $0,871(\mathrm{KMO}>0,5)$ y un contraste de esfericidad con una significancia de $0,000(\mathrm{p}<$ $0,05)$, más tarde, los ítems se discriminan en tres factores; ambos resultados muestran una adecuada validez del instrumento. La prueba Alfa de Cronbach para los 13 ítems obtuvo un resultado de 0,861 , confirmando la hipótesis de confiabilidad del instrumento. Así mismo, la prueba Alfa de Cronbach si se elimina el elemento, obtuvo valores por encima del 0,78 para los 13 ítems.

De la misma forma, el análisis factorial del Cuestionario de barreras para la búsqueda de ayuda para problemas de salud mental, versión breve (BASH-B) obtuvo un índice de adecuación muestral de $0,742(\mathrm{KMO}>0,5)$ y un contraste de esfericidad con una significancia de 0,001 ( $p<$ 0,05 ); ambos resultados muestran una validez adecuada del instrumento. La prueba Alfa de Cronbach para los 10 ítems obtuvo un resultado de 0,790 , confirmando la hipótesis de confiabilidad del instrumento. Así mismo, la prueba Alfa de Cronbach si se elimina el elemento, obtuvo valores por encima del 0,7 para los 10 ítems.

La Tabla 1 presenta los estadísticos descriptivos de la variable autoeficacia académica y sus dimensiones. El puntaje total de la variable Autoeficacia académica, 67 estudiantes $(51,15 \%)$ presentan un índice regular de autoeficacia, seguidos en cantidad por los de índice alto y, por último, los de bajo índice. Con respecto a sus dimensiones, el comportamiento de sus resultados es similar.

Tabla 1. Descriptivos de Autoeficacia Académica

\begin{tabular}{lrrrrr}
\hline & Comunicación & Atención & Excelencia & \multicolumn{1}{c}{ TOTAL } \\
\hline $\mathbf{N}^{\circ}$ de ítems & 4 & 5 & 4 & 13 \\
\hline Rango & $4-20$ & $5-25$ & $4-20$ & $13-65$ \\
\hline Media & 15,24 & 17,78 & 14,98 & 62,03 \\
\hline Desviación típica & 3,320 & 2,932 & 2,877 & 9,180 \\
\hline $\begin{array}{l}\text { (p) Kolmogorov- } \\
\text { Smirnov }\end{array}$ &, 000 &, 000 &, 000 &, 001 \\
\hline Alfa de Cronbach & & & & & \\
\hline & Alta & $40(30,53 \%)$ & $38(29,01 \%)$ & $42(32,06 \%)$ & $40(30,53 \%)$ \\
\cline { 2 - 6 } Rangos & Regular & $72(54,96 \%)$ & $65(49,62 \%)$ & $69(52,67 \%)$ & $67(51,15 \%)$ \\
\cline { 2 - 6 } & Baja & $19(14,50 \%)$ & $28(21,37 \%)$ & $20(15,27 \%)$ & $24(18,32 \%)$ \\
& &, 835 &, 862 &, 861 \\
\hline
\end{tabular}

Fuente: Elaboración propia

Tabla 2. Descriptivos de barreras para la búsqueda de ayuda para problemas de salud mental

\begin{tabular}{lrr}
\hline & \multicolumn{1}{c}{ TOTAL } \\
\hline $\mathrm{N}^{\circ}$ de ítems & 10 \\
\hline Rango & $10-60$ \\
\hline Media & 41,16 \\
\hline Desviación típica & 5,226 \\
\hline (p) Kolmogorov-Smirnov & &, 001 \\
\hline Alfa de Cronbach & &, 790 \\
\hline & Alta & $67(51,15 \%)$ \\
\cline { 2 - 3 } Rangos & Regular & $31(23,66 \%)$ \\
\cline { 2 - 3 } & Baja & $33(25,19 \%)$ \\
\hline
\end{tabular}

Fuente: Elaboración propia 
Tabla 3. Correlaciones

\begin{tabular}{|c|c|c|c|c|c|}
\hline & & $\mathbf{X}$ & $\mathbf{X 1}$ & $\mathbf{X} 2$ & $\mathbf{X 3}$ \\
\hline \multirow{5}{*}{$\begin{array}{l}\text { Correlación } \\
\text { bivariada de } \\
\text { Spearman }\end{array}$} & X. Autoeficacia académica & 1 & & & \\
\hline & X1. Comunicación &, $819^{* *}$ & 1 & & \\
\hline & X2. Atención &, $830^{* *}$ &, $795^{* *}$ & 1 & \\
\hline & X3. Excelencia &, $810^{* *}$ &, $856^{* *}$ & $846^{* *}$ & 1 \\
\hline & Y. Barreras para la búsqueda de ayuda &,$- 358^{*}$ &,$- 402^{*}$ &,$- 312^{*}$ &,$- 337^{*}$ \\
\hline
\end{tabular}

**Correlación significativa al nivel 0,01. *Correlación significativa al nivel 0,05.

Fuente: Elaboración propia.

La Tabla 2 muestra los resultados de la variable barreras para la búsqueda de ayuda para problemas de salud mental. 67 de los casos $(51,15 \%)$ cuenta con una alta presencia de barreras para la búsqueda de ayuda, seguidos en cantidad por los que tienen una presencia baja y regular en proporciones similares.

La distribución de los resultados de las variables y demás componentes resultó no normal $\left(\mathrm{KS}_{(\mathrm{p})}<0,05\right)$, por lo que para el análisis de correlación, se emplearon pruebas no paramétricas.

En la Tabla 3 se exponen los coeficientes de correlación (Rho), de los cuales Autoeficacia académica guarda una fuerte correlación con sus tres componentes. Así mismo, estos componentes guardan relaciones fuertes y significativas entre sí. Sin embargo, la variable autoeficacia académica y sus componentes, guardan una relación significativa inversamente proporcional moderada con la variable barreras para la búsqueda de ayuda para problemas de salud mental.

Los resultados del estudio revelan que, en el caso particular de los estudiantes de una universidad pública de Lima, en su mayoría, los datos tienen una tendencia a los puntajes más altos. El estudio de Valdés Cuervo AA, Cervantes Arreola DI, Dolores Valadez Sierra M, Tánori Quintana J. determinó también que la autoeficacia académica es una variable que tiene a concentrar sus puntajes en las categorías más altas en contextos universitarios (9). El estudio de Manuel García-Fernández J, Inglés-Saura $\mathrm{Cj}$, Vicent $\mathrm{M}$, Gonzálvez C, Lagos-San Martín N, Miguel PérezSánchez A. la distribución de los resultados es muy similar a la obtenida en el presente estudio: el grueso de la población se encuentra en un nivel medio o regular (10). En la investigación de Domínguez-Lara S, Sánchez-Carreño K. la variable Autoeficacia tendría relación con otros elementos como el afrontamiento y regulación emocional, ambas actitudes posee relaciones significativa al igual que las variables del presente estudio (11).

Por último, la desaparición de las barreras para la búsqueda de ayuda para problemas de salud mental puede promoverse desde la sensibilización de la sociedad y las demás estructuras (familia, instituciones públicas, autoridades, prestadores de servicios de salud, etc.) (12). Chau C, y Vilela P, en su estudio sobre determinantes de salud mental en universitarios, se propone que se desarrollen programas que busquen mejorar la salud mental de los universitarios desde múltiples contextos (13). Los problemas de salud mental en universitarios tienen por sintomatologías más frecuentes a las somatizaciones, las obsesionescompulsiones, la sensitividad interpersonales y hasta la depresión (14), sin embargo, el efecto de estos males perjudicaría también el quehacer académico (15). Es importante que la vida académica y la salud mental tengan un tratamiento conjunto por parte de las autoridades de las instituciones educativas de nivel superior. La propuesta de incluir como parte del bienestar del estudiantado estrategias para proteger su salud mental mediante actividades fuera del programa, podría tener un efecto positivo en la eficacia, el desempeño y otras actitudes de beneficio personal para el estudiante y, como resultado, para su formación profesional.

\section{CONCLUSIONES}

Existe una relación significativa inversamente proporcional moderada entre la autoeficacia académica y las barreras para la búsqueda de ayuda para problemas de salud mental en un grupo de estudiantes de una universidad pública de Lima. De la misma forma, el análisis determinó que existe una relación significativa inversamente proporcional moderada entre los componentes Comunicación, Atención y Excelencia de la variable autoeficacia académica y barreras para la 
búsqueda de ayuda para problemas de salud mental en una muestra de universitarios de Lima.

\section{AUTOR DE CORRESPONDENCIA}

Alberto Salvador Palacios Jimenez

EP de Educación

Universidad Nacional Mayor de San Marcos

Dirección:

Teléfono:

\section{REFERENCIAS BIBLIOGRÁFICAS}

1. Dominguez S. Autoeficacia para situaciones académicas en estudiantes universitarios peruanos: un enfoque de ecuaciones estructurales. Rev Psicol - UCSP. 2014;4:45-53 .

2. Bandura. A. Self-Efficacy in changind societies. Cambridge, Cambridge University Press. 1997a.

3. Tsholofeo, A. The relationship between ethbicidenty, collective self-esteem and academic self-efficay among students at a higher learning institution. Requirements for the Degree MA Research Psycology. Departament of Psycology Faculty of Humanities University of Pretoria. 2010.

4. Fernández-Arata $\mathrm{M}$, et al. Ítem único de burnout académico y su relación con autoeficacia académica en estudiantes universitarios. Enferm Clin. 2016.

5. Marco Criolloa, Marcos Romeroa y Tomás Fontaines-Ruiz B. Autoeficacia para el aprendizaje de la investigación en estudiantes universitarios. Psicología Educativa [Internet] 2017 [cited 2018 Oct 8]; 23 (2017) 63-72.

6. Olivari, C. Adaptación y validación del cuestionario de barreras para la búsqueda de ayuda para problemas de salud mental en adolescentes chilenos. Universidad Católica del Maule, Chile [Internet] 2017 [cited 2018 Oct 9].

7. Blanco Vega H, Ornelas Contreras $\mathrm{M}$, Aguirre Chávez Jf, Guedea Delgado Jc. Autoeficacia Percibida En Conductas Académicas: Diferencias entre hombres y mujeres. Revista Mexicana de Investigación Educativa [Internet]. 2012 Apr [cited 2018 Oct 5];17(53):557-71.

8. Wilson, C. J., Deane, F. P., Marshall, K. L., \& Dalley, A. (2008). Reducing adolescents' perceived barriers to treatment and increasing help-seeking intentions: effects of classroom presentations by general practitioners. Journal of Youth and Adolescence, 37(10), 1257-1269.

9. Valdés Cuervo AA, Cervantes Arreola DI, Dolores Valadez Sierra M, Tánori Quintana J. Diferencias en autoeficacia académica, bienestar psicológico y motivación al logro en estudiantes universitarios con alto y bajo desempeño académico. Psicología desde el Caribe [Internet]. 2018 Jan [cited 2018 Oct 10];35(1):39-52.

10. MANUEL GARCÍA-FERNÁNDEZ J, INGLÉS-SAURA CJ, VICENT M, GONZÁLVEZ C, LAGOS-SAN MARTÍN N, MIGUEL PÉREZ-SÁNCHEZ A. Relación entre autoeficacia y autoatribuciones académicas en estudiantes chilenos. Universitas Psychologica [Internet]. 2016 Jan [cited 2018 Oct 17];15(1):79-88.

11. DOMÍNGUEZ-LARA $\mathrm{S}$, SÁNCHEZCARREÑO K. Uso De Estrategias Cognitivas De Regulación Emocional Ante La Desaprobación De Un Examen: El Rol De La Autoeficacia Académica en Estudiantes Universitarios. Psychologia [Internet]. 2017 Jul [cited 2018 Oct 10];11(2):99-111.

12. Campo-Arias A, Oviedo HC, Herazo E. Estigma: barrera de acceso a servicios en salud mental. Revista Colombiana de Psiquiatria [Internet]. 2014 Jul [cited 2018 Oct 17];43(3):162-7.

13. Chau C, Vilela P. Determinantes de la salud mental en estudiantes universitarios de Lima y Huánuco. Psicología (02549247) [Internet]. 2017 Jul [cited 2018 Oct 9];35(2):387-422.

14. Marenco Escuderos A, Suárez Colorado Y, Palacio Sañudo J. Burnout Académico Y Síntomas Relacionados Con Problemas De Salud Mental en Universitarios Colombianos. Psychologia [Internet]. 2017 Jul [cited 2018 Oct 7];11(2):45-55.

15. Pérez Villalobos C, Maldonado García P, Aguilar Aguilar C, Acosta Pérez M. Clima Educativo Y Su Relación Con La Salud Mental De Alumnos Universitarios Chilenos. Revista Argentina de Clínica Psicológica [Internet]. 2013 Nov [cited 2018 Oct 7];22(3):257-68. 